\title{
Review of: "Screening of unruptured intracranial aneurysms in 50 to 60-year-old female smokers: a pilot study"
}

\author{
Carmelo Lucio Sturiale
}

Potential competing interests: The author(s) declared that no potential competing interests exist.

In this pilot study, the Authors proposed the screening of a selected cohort of patients deemed, from previous investigations, as a high risk for unruptured intracranial aneurysms (ulAs) incidence, which are 50 to 60-year-old female smokers.

These patients, extracted from a larger cohort used for a prospective observational study on genetic risk factors for cardiovascular disease, were asked to fill in questionnaires regarding general social and health domains and health-related quality of life, and subsequently, they underwent a computed tomography angiography (CTA) as a screening for ulAs. This cohort was characterized by two unmodifiable risk factors, namely sex and age, and one modifiable that is the smoke habit.

For these patients, therefore, a precocious diagnosis of an ulAs could entail a strong recommendation to quit smoking and change lifestyle, possibly modifying the natural history of an ulA thus reducing the risk of $\mathrm{SAH}$.

As a consequence of their screening on 43 candidates, the Authors overall detected 5 aneurysms in 5 patients (11.6\%) and 1 dural artero-venous fistula in 1 patient (2.3\%). Among the patients harboring ulAs, 1 underwent preventive surgery, while the remaining 4 were treated conservatively with smoking cessation and strict follow-up.

Due to the recent advancements and the increasing spread of neuroimaging, the incidental diagnosis of ulAs has been in general raised in the general population. At the same time, the natural history of these vascular lesions is not fully understood, and it is not exactly predictable, and in the next future, it is difficult to expect further high-quality information about natural history, both for practical and ethical reasons.

Nowadays, neurosurgeons around the world are daily coping with two apparent paradoxes regarding IAs: firstly, the prevalence of ulAs appears higher than the incidence of SAH; secondly, most ruptured intracranial aneurysms (rIAs) are smaller than $5 \mathrm{~mm}$, although the ISAT trial set the risk of rupture cutoff at $7 \mathrm{~mm}$. 
On this ground, the scientific literature demonstrated an increasing interest in trying to preventive detect the unknown ulAs besides those daily incidentally discovered and assess which have a higher risk of rupture. For this purpose, different predicting risk scores for growth and rupture assessment, such as ELAPSS and PHASES, have been proposed. Anyway, none of these takes into consideration smoking as a risk factor and none was replicated as fully reliable in clinical practice in the following validation studies.

However, different arguments may stand against a large screening program for ulAs detection.

First, most ulAs that are detected during the screening would be of small size, a finding confirmed in this index study where 4 out of 5 aneurysms measured less than $5 \mathrm{~mm}$. Notwithstanding, it is known that even small aneurysms can grow and eventually rupture over time. Hence, the cost-benefit of the screening still appears favorable taking into account the burden of the complication of SAH, which still represents a catastrophic event, with high morbidity and mortality rates, despite all the treatment advancements.

Secondly, the psychological impact for the patients who discovered having an ulA can negatively impact their quality of life. In fact, considering the uncertainty in the natural history of such vascular lesions, the personal choice to face the risk of the natural history of the disease or that of the treatment remains a sword of Damocles for patients. In this regard, the most recent UIATS score, belonging to a second score family conceived to produce straightforward recommendations about treatment rather than estimations of growth/rupture risk, set smoke habits and the reduced quality of life due to the fear for aneurysm rupture as variables favoring the uIAs treatment recommendation. These were not taken into consideration in the previous score systems assessing the risk of rupture. Thus, how to deal with the psychological aspects of large-scale aneurysm screening should be investigated in parallel to the advantages in the establishment of a preventive treatment protocol for many of the incidentally discovered aneurysms.

A third problem is represented by the age range considered with the highest ulAs incidence. Interestingly, age is contemplated in all risk score systems, albeit with different thresholds, ranging from 40 to 70 years. With the advent of the fifth decade, however, we can also consider the onset of hypertension as an additional risk factor in predisposed patients.

In conclusion, due to the severity of the hemorrhagic onset of an IA, a large screening program in a higher risk patient population may represent a future desirable approach in those countries with high socioeconomic standards, which can bear the economic burden in their health system. However, in light of the currently limited possibility of predicting natural history despite an accurate assessment of all the available risk variables, we believe that future efforts should be aimed to parallel reduce the treatment-related risks so that a safe treatment can be offered even to patients with an estimated low risk with an incidental or 
post-screening discovered ulA.

Carmelo Lucio Sturiale, MD, PhD, MSc and Vito Stifano, MD.

Fondazione Policlinico Universitario A. Gemelli IRCCS

Università Cattolica del Sacro Cuore, Rome, Italy. 